\title{
Schrader kritisiert Bürokratie und Zentralisierung
}

\author{
"Qualität stellen wir durch Bürokratie sicher", sagte Bernd Heinemann, gesundheitspolitischer \\ Sprecher der Schleswig-Holsteinischen SPD-Landtagsfraktion bei der Podiumsdiskussion des \\ diesjährigen Ostseesymposiums in Kiel. Unter den rund 50 Zahnärztinnen und Zahnärzten im \\ Auditorium sorgte er damit zuerst für ungläubiges Erstaunen und dann für Heiterkeit, als er die- \\ se Aussage damit rechtfertigte, dass die Bürger dies von der Politik erwarteten.
}

In seiner Replik verdeutlichte der Bundesvorsitzende des Freien Verbandes Deutscher Zahnärzte (FVDZ), Harald Schrader, den bürokratischen Wahnsinn, unter dem die Praxen zu leiden haben am Beispiel des fünften Sozialgesetzbuches (SGB V). Zum Zeitpunkt seiner Verpflichtung als damaliger Kassenzahnarzt habe es den Umfang einer Schulkladde gehabt. „Jetzt ist es ein Wälzer mit hunderten von Seiten und von einem Sozialgesetzbuch zu einer kleinteiligen Betriebsanweisung mutiert“, kritisierte Schrader. Hier gebe es dringenden Korrekturbedarf. (Anm. d. Red.: Die aktuelle Ausgabe des SGB V-Handbuchs umfasst 654 Seiten.)

Das eigentliche Thema der Podiumsdiskussion „MVZ - Teil der Lösung oder Teil des Problems“ geriet dabei fast ein wenig in den Hintergrund. Unter Moderation des FVDZ-Landesvorsitzenden Dr. Joachim Hüttmann diskutierten die gesundheitspolitischen Sprecher ihrer Fraktionen im Schleswig-Holsteinischen Landtag Karsten Jasper (CDU), Bernd Heinemann (SPD) Dr. Heiner Garg (FDP) Wolfgang Dudda (Piraten) und der FVDZ-Bundesvorsitzende Schrader.

\section{Arztgleiches MVZ ist Betriebsunfall}

In einer kurzen Einführung hatte zunächst der Justiziar des Freien Verbandes, Michael Lennartz, die Thematik zahnmedizinischer Versorgunszentren beleuchtet. Dabei wies er besonders auf die Folgen der Streichung des Wortes „fachübergreifend“ im GKV-Versorgungsstärkungsgesetz hin. Das arztgleiche MVZ sei für Ärzte durchaus sinnvoll, erweise sich für Zahnärzte jedoch eher als Betriebsunfall. Insbesondere die Möglichkeit für Kommunen, ein MVZ zu betreiben, bezeichnete Lennartz als Sündenfall. Damit werde dem bislang verbotenen Fremdbesitz Tür und Tor geöffnet. Im MVZ beliebig viele angestellte Zahnärzte beschäftigen zu können, verletze die Chancengleichheit gegenüber anderen Berufsausübungsgemeinschaften eklatant, betonte der Rechtsanwalt. Für diese gelte weiterhin die Beschränkung auf maximal zwei angestellte Zahnärzte. So stelle sich die Frage nach der Intention des Gesetzgebers. Für Lennartz gibt es nur eine Interpretation: „Die MVZ sind ein weiterer Baustein auf dem Weg zu Selektivverträgen mit Krankenkassen und privaten Krankenversichern, an dessen Ende die Industrialisierung des Gesundheitssystems steht.“

Dieser Einschätzung schloss sich der FDP-Abgeordnete Garg an. Die ehemalige Bundesgesundheitsministerin Ulla Schmidt (SPD) habe alles daran gesetzt, die Freiberuflichkeit zu schwächen, und MVZ seien ein weiterer Schritt in diese Richtung. Es sei Humbug anzunehmen, irgendjemand würde freiwillig ein MVZ gründen, um den ländlichen Raum besser zu versorgen. Der SPD-Vertreter Heinemann hingegen vertrat die Ansicht, wenn 70 Prozent der Studierenden Frauen mit einer ganz anderen Vorstellung von Work-Life-Balance seien, dann seien diese nicht „so blöd“, sich auch noch eine eigene Praxis ans Bein zu binden. Deshalb müsse die Politik Alternativen ermöglichen. CDU-Mann Jasper räumte ein, dass die Anzahl möglicher angestellter Zahnärzte in MVZ unbedingt begrenzt werden müsse. Auf den Punkt brachte es schließlich Dudda als Vertreter der Piraten, indem er feststellte, unbegrenzte MVZ seien keine Lösung, sondern das Problem.

\section{Kollateralschaden der Ärzte}

Der FVDZ-Bundesvorsitzende Schrader sprach dem gesamten Auditorium aus dem Herzen, als er beklagte, dass die Zahnärzteschaft bei gesetzlichen Neuregelungen immer als Kollateralschaden der Ärzte durchginge. Bei den jungen Kolleginnen und Kollegen sei durchaus Bereitschaft vorhanden, sich auch im ländlichen Raum niederzulassen. Allerdings saugten die MVZ mit ihren Anstellungsangeboten genau diese bereitwilligen Kolleginnen und Kollegen ab und verschärften damit Versorgungsprobleme. „Auch deshalb führt die zunehmende Ökonomisierung des Gesundheitswesens keinesfalls zu einer Verbesserung der Versorgung“, betonte Schrader. Hinter dem von der Politik so gern benutzten Begriff der Deregulierung habe sich bisher immer nur eine weitere Zentralisierung versteckt. Wenn es die Politik wirklich ernst meine mit neuen Versorgungsformen, so Schraders Forderung, dann könne man beispielsweise genossenschaftlich organisierte Versorgungsformen ermöglichen.

Dr. Holger Neumeyer

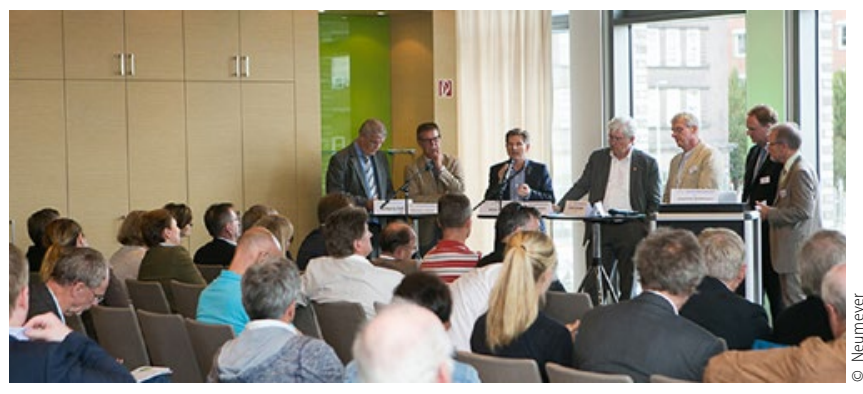

Hochkarätiges Podium aus der Landespolitik beim Ostseesymposium 